\title{
QUADRATIC MALCEV SUPERALGEBRAS WITH REDUCTIVE EVEN PART
}

\author{
HELENA ALBUQUERQUE, ELISABETE BARREIRO, AND SAÏD BENAYADI
}

\begin{abstract}
It is our goal to give an inductive description of quadratic Malcev superalgebras with reductive even part. We use the notion of double extension of Malcev superalgebras presented by $\mathrm{H}$. Albuquerque and S. Benayadi in [4] and transfer to Malcev superalgebras the concept of generalized double extension given in [5] for Lie superalgebras.
\end{abstract}

\section{INTRODUCTION}

In this work all the quadratic Malcev superalgebras considered are finite dimensional, defined over an algebraically closed field of characteristic 0 . The description of Malcev superalgebras with reductive even part and the action of the even part on the odd part is completely reducible was reduced in 1 to the classification of Lie superalgebras satisfying the same conditions. This problem was solved by Alberto Elduque in [11, inside the description of the structure of semisimple Lie superalgebras using the notions of nice extension and elementary extension.

A quadratic Malcev superalgebra is a Malcev superalgebra where is defined an even non-degenerate invariant supersymmetric bilinear form. Quadratic Lie algebras were classified by A. Medina and P. Revoy in [12 using the notion of double extension of Lie algebras. This notion was extended in $[6]$ to Lie superalgebras and S. Benayadi, in [7, has used this definition to study Lie superalgebras with reductive even part and the action of the even part on the odd part is completely reducible having these results been generalized in 4 for Malcev superalgebras.

In case that we do not demand that the action of the even part on the odd part is completely reducible we have a larger class of Lie superalgebras with reductive even part which contain the preceeding ones and the remaining examples that appear in this study, obliged us to use the notion of generalized double extension for Lie superalgebras introduced in $[5$ with the purpose to give an inductive description of solvable quadratic Lie superalgebras.

In this work we generalize all the results mentioned before, presenting an inductive description of Malcev superalgebras with reductive even part. We prove that if $\mathfrak{U}$ is the set formed by $\{0\}$, the basic classical simple Lie superalgebras, the non Lie simple Malcev algebra and the one-dimensional Lie algebra, every Malcev superalgebra with reductive even part is either an element of $\mathfrak{U}$ or is obtained from a finite number of elements of $\mathfrak{U}$ by a sequence of double extensions by the one dimensional Lie algebra or/and generalized double extensions by the one dimensional Lie superalgebra with null even part or/and orthogonal direct sums.

Key words and phrases. Malcev superalgebras; Reductive Malcev algebras; Quadratic forms.

The first and the second authors acknowledge partial financial assistance by the CMUC, Department of Mathematics, University of Coimbra. 


\section{BASIC NOTIONS}

For basic notions and properties concerning quadratic Malcev superalgebras we propose to the reader a careful lecture of the [1, 4. In this paper, we shall consider finite dimensional Malcev superalgebras over an algebraically closed commutative field $\mathbb{K}$ of characteristic zero.

Definition 1.1. A superalgebra $M=M_{\overline{0}} \oplus M_{\overline{1}}$ (meaning a $\mathbb{Z}_{2}$-graded algebra) is called a Malcev superalgebra if $X Y=-(-1)^{x y} Y X, \forall X \in M_{x}, Y \in M_{y}$, and

$$
\begin{aligned}
(-1)^{y z}(X Z)(Y T)= & ((X Y) Z) T+(-1)^{x(y+z+t)}((Y Z) T) X \\
& +(-1)^{(x+y)(z+t)}((Z T) X) Y+(-1)^{t(x+y+z)}((T X) Y) Z,
\end{aligned}
$$

for $X \in M_{x}, Y \in M_{y}, Z \in M_{z}$, and $T \in M_{t}$. We shall write $X \in M_{x}$ to mean that $X$ is a homogeneous element of the Malcev superalgebra $M$ of degree $x$, with $x \in \mathbb{Z}_{2}$.

Definition 1.2. Let $M$ be a Malcev superalgebra and $\phi: M \longrightarrow M$ an endomorphism of $M$. We say that $\phi$ is a Malcev operator of $M$ if

$$
\begin{aligned}
\phi((X Y) Z)= & (\phi(X) Y) Z-(-1)^{x y} \phi(Y)(X Z)-(-1)^{z(x+y)}(\phi(Z) X) Y \\
& -(-1)^{x(y+z)} \phi(Y Z) X, \quad \forall X \in M_{x}, Y \in M_{y}, Z \in M_{z} .
\end{aligned}
$$

We denote by $(O p(M))_{\alpha}$ the vector subspace of $\operatorname{End}(M)$ formed by the Malcev operators of $M$ of degree $\alpha\left(\alpha \in Z_{2}\right)$. Then $O p(M)=(O p(M))_{\overline{0}} \oplus(O p(M))_{\overline{1}}$.

Definition 1.3. Let $M$ be a Malcev superalgebra. A bilinear form $B$ on $M$ is called

(i) supersymmetric if $B(X, Y)=(-1)^{x y} B(Y, X), \forall X \in M_{x}, Y \in M_{y}$.

(ii) non-degenerate if $X \in M$ satisfies $B(X, Y)=0, \forall_{Y \in M}$, then $X=0$.

(iii) invariant if $B(X Y, Z)=B(X, Y Z), \forall X, Y, Z \in M$.

(iv) even if $B(X, Y)=0, \forall(X, Y) \in M_{\alpha} \times M_{\beta},(\alpha, \beta) \in \mathbb{Z}_{2} \times \mathbb{Z}_{2}(\alpha \neq \beta)$.

Definition 1.4. A Malcev superalgebra $M$ is called quadratic if there exists a bilinear form $B$ on $M$ such that $B$ is even, supersymmetric, non-degenerate, and invariant. It is denoted by $(M, B)$ and $B$ is called an invariant scalar product on $M$.

Definition 1.5. Let $(M, B)$ be a quadratic Malcev superalgebra. A homogeneous $\operatorname{map} \phi \in \operatorname{End}(M)$ of degree $\alpha$ (with $\alpha \in \mathbb{Z}_{2}$ ) is called skew-supersymmetric if

$$
B(\phi(X), Y)=-(-1)^{\alpha x} B(X, \phi(Y)), \quad \forall X \in M_{x}, Y \in M .
$$

We denote by $\left(O p_{a}(M)\right)_{\alpha}$ the vector subspace of the skew-supersymmetric elements of $(O p(M))_{\alpha}$. We write $O p_{a}(M)=\left(O p_{a}(M)\right)_{\overline{0}} \oplus\left(O p_{a}(M)\right)_{\overline{1}}$ which is a super-vector subspace of $O p(M)$.

Definition 1.6. Let $M$ be a Malcev superalgebra and $V$ a $\mathbb{Z}_{2}$-graded vector space. Let $\omega: M \times M \longrightarrow V$ be a homogeneous bilinear map. If the assertion $\omega(X, Y)=$ $-(-1)^{x y} \omega(Y, X), \forall X \in M_{x}, Y \in M_{y}$, and

$$
\begin{aligned}
(-1)^{y z} \omega(X Z, Y T)= & \omega((X Y) Z, T)+(-1)^{x(y+z+t)} \omega((Y Z) T, X) \\
& +(-1)^{(x+y)(z+t)} \omega((Z T) X, Y) \\
& +(-1)^{t(x+y+z)} \omega((T X) Y, Z), \quad \forall_{X \in M_{x}, Y \in M_{y}, Z \in M_{z}, T \in M_{t},}
\end{aligned}
$$

are satisfied we say that $\omega$ is a Malcev 2-cocycle on $M$ with values in $V$. 
Proposition $1.7([4)$. Let $(M, B)$ be a quadratic Malcev superalgebra and $\omega$ : $M \times M \longrightarrow \mathbb{K}$ a bilinear form of degree $\alpha \in \mathbb{Z}_{2}$.

(i) There exists a homogeneous map $\phi \in \operatorname{End}(M)$ of degree $\alpha$ such that

$$
\omega(X, Y)=B(\phi(X), Y), \quad \forall X, Y \in M .
$$

(ii) $\omega$ is a Malcev 2-cocycle on $M$ if and only if $\phi$ is a skew-supersymmetric Malcev operator of $M$.

\section{Generalized double extension of QuAdratic MalceV superalgebras}

We consider $\mathbb{K} e=(\mathbb{K} e)_{\overline{1}}$ the one-dimensional abelian Malcev superalgebra with even part zero and $\mathbb{K} e^{*}$ its dual vector space.

Proposition 2.1. Let $(M, B)$ be a quadratic Malcev superalgebra and $D$ an odd skew-supersymmetric Malcev operator of $(M, B)$. Let us consider the bilinear map $\varphi: M \times M \longrightarrow \mathbb{K} e^{*}$ defined by

$$
\varphi(X, Y)=-B(D(X), Y) e^{*}, \quad \forall X, Y \in M .
$$

Then $\varphi$ is a Malcev 2-cocycle on $M$ with values in $\mathbb{K} e^{*}$. Moreover, the $\mathbb{Z}_{2}$-graded vector space $M \oplus \mathbb{K} e^{*}$ with the multiplication defined by

$$
\left(X+\alpha e^{*}\right)\left(Y+\beta e^{*}\right)=X Y+\varphi(X, Y), \quad \forall\left(X+\alpha e^{*}\right),\left(Y+\beta e^{*}\right) \in\left(M \oplus \mathbb{K} e^{*}\right),
$$

is a Malcev superalgebra that is called the central extension of $\mathbb{K} e^{*}$ by $M$ (by means of $\varphi$ ).

Proof. We will show that $M \oplus \mathbb{K} e^{*}$ is a Malcev superalgebra. Since $D$ is odd and skew-supersymmetric we have that $\varphi(X, Y)=-(-1)^{x y} \varphi(X, Y)$, for all $X \in$ $M_{x}, Y \in M_{y}$. As the multiplication in $M$ is graded skew-symmetric we conclude that also the multiplication defined in $M \oplus \mathbb{K} e^{*}$ is graded skew-symmetric. We have to ensure that

$$
\begin{aligned}
(-1)^{y z}(( & \left.\left.X+\alpha e^{*}\right)\left(Z+\gamma e^{*}\right)\right)\left(\left(Y+\beta e^{*}\right)\left(T+\delta e^{*}\right)\right)= \\
= & \left(\left(\left(X+\alpha e^{*}\right)\left(Y+\beta e^{*}\right)\right)\left(Z+\gamma e^{*}\right)\right)\left(T+\delta e^{*}\right) \\
& +(-1)^{x(y+z+t)}\left(\left(\left(Y+\beta e^{*}\right)\left(Z+\gamma e^{*}\right)\right)\left(T+\delta e^{*}\right)\right)\left(X+\alpha e^{*}\right) \\
& +(-1)^{(x+y)(z+t)}\left(\left(\left(Z+\gamma e^{*}\right)\left(T+\delta e^{*}\right)\right)\left(X+\alpha e^{*}\right)\right)\left(Y+\beta e^{*}\right) \\
& +(-1)^{t(x+y+z)}\left(\left(\left(T+\delta e^{*}\right)\left(X+\alpha e^{*}\right)\right)\left(Y+\beta e^{*}\right)\right)\left(Z+\gamma e^{*}\right),
\end{aligned}
$$

$\forall\left(X+\alpha e^{*}\right) \in\left(M \oplus \mathbb{K} e^{*}\right)_{x},\left(Y+\beta e^{*}\right) \in\left(M \oplus \mathbb{K} e^{*}\right)_{y},\left(Z+\gamma e^{*}\right) \in\left(M \oplus \mathbb{K} e^{*}\right)_{z},\left(T+\delta e^{*}\right) \in\left(M \oplus \mathbb{K} e^{*}\right)_{t}$, or equivalently,

$$
\begin{aligned}
(-1)^{y z}\{( & X Z)(Y T)+\varphi(X Z, Y T)\}= \\
= & ((X Y) Z) T+\varphi((X Y) Z, T) \\
& +(-1)^{x(y+z+t)}\{((Y Z) T) X+\varphi((Y Z) T, X)\} \\
& +(-1)^{(x+y)(z+t)}\{((Z T) X) Y+\varphi((Z T) X, Y)\} \\
& +(-1)^{t(x+y+z)}\{((T X) Y) Z+\varphi((T X) Y, Z)\} .
\end{aligned}
$$

$\forall\left(X+\alpha e^{*}\right) \in\left(M \oplus \mathbb{K} e^{*}\right)_{x},\left(Y+\beta e^{*}\right) \in\left(M \oplus \mathbb{K} e^{*}\right)_{y},\left(Z+\gamma e^{*}\right) \in\left(M \oplus \mathbb{K} e^{*}\right)_{z},\left(T+\delta e^{*}\right) \in\left(M \oplus \mathbb{K} e^{*}\right)_{t}$. Since $B$ is non-degenerate and $D$ is a Malcev operator then we have

$\forall\left(X+\alpha e^{*}\right) \in\left(M \oplus \mathbb{K} e^{*}\right)_{x},\left(Y+\beta e^{*}\right) \in\left(M \oplus \mathbb{K} e^{*}\right)_{y},\left(Z+\gamma e^{*}\right) \in\left(M \oplus \mathbb{K} e^{*}\right)_{z},\left(T+\delta e^{*}\right) \in\left(M \oplus \mathbb{K} e^{*}\right)_{t}$,

$$
\begin{aligned}
(-1)^{y z} \varphi(X Z, Y T)= & \varphi((X Y) Z, T)+(-1)^{x(y+z+t)} \varphi((Y Z) T, X) \\
& +(-1)^{(x+y)(z+t)} \varphi((Z T) X, Y) \\
& +(-1)^{t(x+y+z)} \varphi((T X) Y, Z) .
\end{aligned}
$$


We infer that $\varphi$ is a Malcev 2-cocycle of $M$ with values in $\mathbb{K} e^{*}$. Using the fact that $M$ is a Malcev superalgebra we conclude that $M \oplus \mathbb{K} e^{*}$ is a Malcev superalgebra as required.

Proposition 2.2. Consider two Malcev superalgebras $M$ and $V$, a linear map $\Omega: M \longrightarrow O p(V)$, and an even skew-supersymmetric bilinear map $\zeta: M \times M \longrightarrow V$ such that

(i) $\forall_{X \in M_{x}, Y \in M_{y}, h \in V_{z}, i \in V_{t}}$,

$$
\begin{aligned}
& (\Omega(X Y)(h)) i-\Omega(X)(\Omega(Y)(h) i)-(-1)^{y z}(\Omega(X)(h))(\Omega(Y)(i)) \\
& +(-1)^{x y} \Omega(Y)(\Omega(X)(h i))+(-1)^{t z+x y} \Omega(Y)(\Omega(X)(i)) h \\
& \quad+(\zeta(X, Y)(h)) i=0 ;
\end{aligned}
$$

(ii) $\forall X \in M_{x}, Z \in M_{z}, g \in V_{y}, i \in V_{t}$,

$$
\begin{aligned}
(-1)^{y z}\{\zeta(X, Z) & (g i)+\Omega(X Z)(g i)\}=-(-1)^{z(x+y)} \Omega(Z)(\Omega(X)(g)) i \\
& +(-1)^{y z} \Omega(X)(\Omega(Z)(g) i)-(-1)^{y(z+t)} \Omega(X)(\Omega(Z)(i)) g \\
& +(-1)^{t y+(x+y) z} \Omega(Z)(\Omega(X)(i) g) ;
\end{aligned}
$$

(iii) $\forall X \in M_{x}, Y \in M_{y}, T \in M_{t}, h \in V_{z}$,

$$
\begin{gathered}
(-1)^{y z}(\Omega(X)(h)) \zeta(Y, T)+(-1)^{t(x+y+z)} \Omega(T)(\zeta(X, Y) h) \\
+(-1)^{t(x+z)+x y} \Omega(Y)(\zeta(T, X)) h=0
\end{gathered}
$$

(iv) $\forall X \in M_{x}, Y \in M_{y}, Z \in M_{z}, T \in M_{t}$,

$$
\begin{aligned}
& -(-1)^{y z} \Omega(X Z)(\zeta(Y, T))+(-1)^{t(y+z)} \Omega(X)(\Omega(T)(\zeta(Y, Z))) \\
& +(-1)^{x(y+z+t)+y z} \Omega(Z)(\Omega(Y)(\zeta(T, X)))-\Omega(X)(\zeta(Y Z, T)) \\
& -(-1)^{(x+y)(z+t)} \Omega(Z)(\zeta(T X, Y))= \\
& =-(-1)^{y z}\{\zeta(X Z, Y T)+\zeta(X, Z) \zeta(Y, T)\}+\zeta((X Y) Z, T) \\
& \quad+(-1)^{x(y+z+t)} \zeta((Y Z) T, X)+(-1)^{(x+y)(z+t)} \zeta((Z T) X, Y) \\
& \quad+(-1)^{t(x+y+z)} \zeta((T X) Y, Z) ;
\end{aligned}
$$

(v) $\forall_{X \in M_{x}, Y \in M_{y}, Z \in M_{z}, i \in V_{t}}$,

$$
\begin{aligned}
(-1)^{y z} \Omega( & X Z)(\Omega(Y)(i))-\zeta(X Y, Z) i-\Omega((X Y) Z)(i) \\
& +\Omega(X)(\Omega(Y Z)(i))-(-1)^{x y} \Omega(Y)(\Omega(X)(\Omega(Z)(i))) \\
& +(-1)^{(x+y) z+x y} \Omega(Z)(\Omega(Y)(\Omega(X)(i)))=0 .
\end{aligned}
$$

Then the $\mathbb{Z}_{2}$-graded vector space $M \oplus V$ endowed with the multiplication

$$
(X+f)(Y+h)=(X Y)_{M}+(f h)_{V}+\Omega(X)(h)-(-1)^{x y} \Omega(Y)(f)+\zeta(X, Y)
$$

where $(X+f) \in(M \oplus V)_{x}$ and $(Y+h) \in(M \oplus V)_{y}$, is a Malcev superalgebra that is called the generalized semi-direct product of $M$ and $V$ (by means of $\Omega$ and $\zeta$ ). In particular, if $\zeta=0$ then we have the semi-direct product of $M$ and $V$ (by means of ת) 4 .

Proof. It is analogous to the proof of the [4, Theorem 3.1]. To show that $M \oplus V$ is a Malcev superalgebra it is suffices to see the axiom of definition of a Malcev superalgebra with $((X Y) Z) T$ in the following cases. If all these elements are in $M$ it is clear since $M$ is a Malcev superalgebra and we have assertion (iv). For the case where all these elements are in $V$ it is direct because $V$ is a Malcev superalgebra. In case where one of these elements is in $M$ and the other three are in $V$ we know that $\Omega(X)$ is a Malcev operator, for all $X \in M$. The conditions (i) and (ii) imply that the axiom is valid in the case $X \in M_{x}, Y \in M_{y}, h \in V_{z}, i \in V_{t}$, 
and on the other hand, the assertions (iii) and (v) show the equality in the case $X \in M_{x}, Y \in M_{y}, Z \in M_{z}, i \in V_{t}$. Conversely, if $M \oplus V$ is a Malcev superalgebra these conditions are verified.

Now, we shall use the particular case of generalized semi-direct product of the Malcev superalgebras $M \oplus \mathbb{K} e^{*}$ and $\mathbb{K} e$ (where $(M, B)$ is a quadratic Malcev superalgebra and $(\mathbb{K} e)_{\overline{1}}$ the one-dimensional abelian Malcev superalgebra with even part zero)

Theorem 2.3. Let $\left(M=M_{\overline{0}} \oplus M_{\overline{1}}, B\right)$ be a quadratic Malcev superalgebra. Suppose that $D \in\left(O p_{a}(M)\right)_{\overline{1}}$ and $A_{0} \in M_{\overline{0}}$ such that

$$
\begin{aligned}
& D^{2}\left(A_{0}\right)=\frac{1}{2} A_{0} A_{0} \\
& D\left(A_{0} X\right)=A_{0} D(X)-D\left(A_{0}\right) X, \\
& \left(A_{0} X\right) Y=D(D(X) Y)+D^{2}(X Y)+(-1)^{x} D(X) D(Y)+(-1)^{x y} D^{2}(Y) X, \\
& A_{0}(X Y)=D(D(X) Y)+D^{2}(X) Y-(-1)^{x y}\left\{D^{2}(Y) X+D(D(Y) X)\right\},
\end{aligned}
$$

where $X \in M_{x}, Y \in M_{y}$ are arbitrary. Define a map $\Omega:(\mathbb{K} e)_{\overline{1}} \longrightarrow O p\left(M \oplus \mathbb{K} e^{*}\right)$ by $\Omega(e)=\widetilde{D}$, where $\widetilde{D}: M \oplus \mathbb{K} e^{*} \longrightarrow M \oplus \mathbb{K} e^{*}$ satisfies $\widetilde{D}\left(e^{*}\right)=0$ and

$$
\widetilde{D}(X)=D(X)-(-1)^{x} B\left(X, X_{0}\right) e^{*}, \quad \forall X \in M_{x} .
$$

Consider the map $\zeta: \mathbb{K} e \times \mathbb{K} e \longrightarrow M \oplus \mathbb{K} e^{*}$ defined by $\zeta(e, e)=A_{0}$. Then $\mathfrak{k}=\mathbb{K} e \oplus M \oplus \mathbb{K} e^{*}$ equipped with the even skew-symmetric bilinear map on $\mathfrak{k}$ defined by

$$
\begin{array}{rlrl}
e e & =A_{0}, & \\
e X & =D(X)-(-1)^{x} B\left(X, A_{0}\right) e^{*}, & & \forall X \in M_{x}, \\
X Y & =(X Y)_{M}-B(D(X), Y) e^{*}, & & \forall X, Y \in M, \\
e^{* \mathfrak{k}} & =\{0\}, &
\end{array}
$$

is the generalized semi-direct product of $M \oplus \mathbb{K} e^{*}$ by the one-dimensional Malcev superalgebra $(\mathbb{K} e)_{\overline{1}}$ (by means of $\Omega$ and $\zeta$ ). Moreover the supersymmetric bilinear form $\widetilde{B}: \mathfrak{k} \times \mathfrak{k} \longrightarrow \mathbb{K}$ defined by

$$
\begin{aligned}
& \left.\widetilde{B}\right|_{M \times M}=B, \\
& \widetilde{B}\left(e, e^{*}\right)=1, \\
& \widetilde{B}(M, e)=\widetilde{B}\left(M, e^{*}\right)=\{0\}, \\
& \widetilde{B}(e, e)=\widetilde{B}\left(e^{*}, e^{*}\right)=0,
\end{aligned}
$$

is an invariant scalar product on $\mathfrak{k}$. The quadratic Malcev superalgebra $(\mathfrak{k}, \widetilde{B})$ is called the generalized double extension of $(M, B)$ by the one-dimensional Malcev superalgebra $(\mathbb{K} e)_{\overline{1}}$ (by means of $D$ and $\left.A_{0}\right)$.

Proof. We start by showing that $\widetilde{D}$ is a Malcev operator of $M \oplus \mathbb{K} e^{*}$. For all $\left(X+\alpha e^{*}\right) \in\left(M \oplus \mathbb{K} e^{*}\right)_{x},\left(Y+\beta e^{*}\right) \in\left(M \oplus \mathbb{K} e^{*}\right)_{y},\left(Z+\gamma e^{*}\right) \in\left(M \oplus \mathbb{K} e^{*}\right)_{z}$ we have to ensure that

$$
\begin{aligned}
\widetilde{D}\left(\left(\left(X+\alpha e^{*}\right)(Y+\right.\right. & \left.\left.\left.\beta e^{*}\right)\right)\left(Z+\gamma e^{*}\right)\right)=\left(\widetilde{D}\left(X+\alpha e^{*}\right)\left(Y+\beta e^{*}\right)\right)\left(Z+\gamma e^{*}\right) \\
& -(-1)^{x y} \widetilde{D}\left(Y+\beta e^{*}\right)\left(\left(X+\alpha e^{*}\right)\left(Z+\gamma e^{*}\right)\right) \\
& -(-1)^{z(x+y)}\left(\widetilde{D}\left(Z+\gamma e^{*}\right)\left(X+\alpha e^{*}\right)\right)\left(Y+\beta e^{*}\right) \\
& -(-1)^{x(y+z)} \widetilde{D}\left(\left(Y+\beta e^{*}\right)\left(Z+\gamma e^{*}\right)\right)\left(X+\alpha e^{*}\right) .
\end{aligned}
$$


Doing easy calculations, this condition is equivalent to

$$
\begin{aligned}
& D((X Y) Z)-(-1)^{x+y+z} B\left((X Y) Z, A_{0}\right) e^{*}= \\
&=(D(X) Y) Z-B(D(D(X) Y), Z) e^{*} \\
& \quad-(-1)^{x y} D(Y)(X Z)+(-1)^{x y} B\left(D^{2}(Y), X Z\right) e^{*} \\
& \quad-(-1)^{z(x+y)}(D(Z) X) Y+(-1)^{z(x+y)} B(D(D(Z) X), Y) e^{*} \\
&-(-1)^{x(y+z)} D(Y Z) X+(-1)^{x(y+z)} B\left(D^{2}(Y Z), X\right) e^{*} .
\end{aligned}
$$

Since $D$ is a Malcev operator, it remains to see that

$$
\begin{aligned}
-B((X Y) Z, & \left.A_{0}\right)=-B(D(D(X) Y), Z)+(-1)^{x y} B\left(D^{2}(Y), X Z\right) \\
& +(-1)^{z(x+y)} B(D(D(Z) X), Y)+(-1)^{x(y+z)} B\left(D^{2}(Y Z), X\right) .
\end{aligned}
$$

As $D$ is skew-supersymmetric and $B$ is non-degenerate, it is the assertion (2.2). Now we have to care about the several conditions of Proposition 2.2. Condition (v) is immediate and (iv) comes direct from $D^{2}\left(A_{0}\right)=\frac{1}{2} A_{0} A_{0}$. We have to verify that for all $\left(Z+\gamma e^{*}\right) \in\left(M \oplus \mathbb{K} e^{*}\right)_{z},\left(T+\delta e^{*}\right) \in\left(M \oplus \mathbb{K} e^{*}\right)_{t}$,

$$
\begin{aligned}
& \left(\Omega(e e)\left(Z+\gamma e^{*}\right)\right)\left(T+\delta e^{*}\right)-\Omega(e)\left(\Omega(e)\left(Z+\gamma e^{*}\right)\left(T+\delta e^{*}\right)\right) \\
& -(-1)^{z}\left(\Omega(e)\left(Z+\gamma e^{*}\right)\right)\left(\Omega(e)\left(T+\delta e^{*}\right)\right)-\Omega(e)\left(\Omega(e)\left(\left(Z+\gamma e^{*}\right)\left(T+\delta e^{*}\right)\right)\right) \\
& -(-1)^{t z} \Omega(e)\left(\Omega(e)\left(T+\delta e^{*}\right)\right)\left(Z+\gamma e^{*}\right)+\left(\zeta(e, e)\left(Z+\gamma e^{*}\right)\right)\left(T+\delta e^{*}\right)=0,
\end{aligned}
$$

which we easily see that it is equivalent to

$$
\begin{aligned}
& -D(D(Z) T)-(-1)^{z} D(Z) D(T)-D^{2}(Z T)-(-1)^{z t} D^{2}(T) Z+\left(A_{0} Z\right) T \\
& \quad-(-1)^{z+t} B\left(D(Z) T, A_{0}\right) e^{*}-(-1)^{z+t} B\left(D(Z T), A_{0}\right) e^{*}-B\left(D\left(A_{0} Z\right), T\right) e^{*} \\
& \quad+(-1)^{z} B\left(D^{2}(Z), D(T)\right) e^{*}+(-1)^{z t} B\left(D\left(D^{2}(T)\right), Z\right) e^{*}=0 .
\end{aligned}
$$

From (2.1) and (2.2), since $B$ is even and non-degenerate, and $D$ is an even skewsupersymmetric map, this expression comes straightforward. Furthermore, we have to prove that for all $\left(Y+\beta e^{*}\right) \in\left(M \oplus \mathbb{K} e^{*}\right)_{y},\left(T+\delta e^{*}\right) \in\left(M \oplus \mathbb{K} e^{*}\right)_{t}$,

$$
\begin{aligned}
(-1)^{y}\{\zeta(e, e)((Y+ & \left.\left.\left.\beta e^{*}\right)\left(T+\delta e^{*}\right)\right)+\Omega(e e)\left(\left(Y+\beta e^{*}\right)\left(T+\delta e^{*}\right)\right)\right\} \\
= & (-1)^{y} \Omega(e)\left(\Omega(e)\left(Y+\beta e^{*}\right)\right)\left(T+\delta e^{*}\right) \\
& +(-1)^{y} \Omega(e)\left(\Omega(e)\left(Y+\beta e^{*}\right)\left(T+\delta e^{*}\right)\right) \\
& -(-1)^{y(1+t)} \Omega(e)\left(\Omega(e)\left(T+\delta e^{*}\right)\right)\left(Y+\beta e^{*}\right) \\
& -(-1)^{y(1+t)} \Omega(e)\left(\Omega(e)\left(T+\delta e^{*}\right)\left(Y+\beta e^{*}\right)\right),
\end{aligned}
$$

doing easy computation, it comes

$$
\begin{aligned}
A_{0}(Y T)-B\left(D\left(A_{0}\right)\right. & , Y T) e^{*}=D^{2}(Y) T-B\left(D^{3}(Y), T\right) e^{*} \\
& +D(D(Y) T)+(-1)^{y+t} B\left(D(Y) T, A_{0}\right) e^{*} \\
& -(-1)^{y t}\left\{D^{2}(T) Y-B\left(D^{3}(T), Y\right) e^{*}\right\} \\
& -(-1)^{y t}\left\{D(D(T) Y)+(-1)^{y+t} B\left(D(T) Y, A_{0}\right) e^{*}\right\} .
\end{aligned}
$$

From (2.1) and (2.3), since $B$ is even and non-degenerate, and $D$ is an even skewsupersymmetric map, we obtain the assertion (ii) of the Proposition 2.2. Finally, we will show that for all $\left(Z+\gamma e^{*}\right) \in\left(M \oplus \mathbb{K} e^{*}\right)_{z}$,

$$
\begin{gathered}
(-1)^{z}\left(\Omega(e)\left(Z+\gamma e^{*}\right)\right) \zeta(e, e)+(-1)^{z} \Omega(e)\left(\zeta(e, e)\left(Z+\gamma e^{*}\right)\right) \\
+(-1)^{z} \Omega(e)(\zeta(e, e))\left(Z+\gamma e^{*}\right)=0,
\end{gathered}
$$

after some calculus, it is

$$
\begin{aligned}
D(Z) A_{0} & +D\left(A_{0} Z\right)+D\left(A_{0}\right) Z \\
& -B\left(D^{2}(Z), A_{0}\right) e^{*}-(-1)^{z} B\left(A_{0} Z ; A_{0}\right) e^{*}-B\left(D^{2}\left(A_{0}\right), Z\right) e^{*}=0 .
\end{aligned}
$$


From (2.1), using $D^{2}\left(A_{0}\right)=\frac{1}{2} A_{0} A_{0}$, since $B$ is even and non-degenerate, and $D$ is skew-supersymmetric we get the desired expression. It is elementary to see that $\mathbb{K} e \oplus M \oplus \mathbb{K} e^{*}$ equipped with multiplication defined by (2.4) is the generalized semi-direct product of $M \oplus \mathbb{K} e^{*}$ by the one-dimensional Malcev superalgebra $(\mathbb{K} e)_{\overline{1}}$ (by means of $\Omega$ and $\zeta$ ).

We use the preceeding notion to present a class of quadratic Malcev superalgebras with reductive even part and the action of the even part on the odd part is not completely reducible

Example 2.4. Let $\left(M=M_{\overline{0}} \oplus M_{\overline{1}}, B\right)$ be the quadratic Malcev superalgebra such that $\operatorname{dim}_{\overline{0}}=1$ and the dimension of $M_{\overline{1}}$ is even. Let $\{a\}$ be a basis of $M_{\overline{0}}$ and $\left\{v_{1}, v_{2}, \ldots, v_{n}, y_{1}, y_{2}, \ldots, y_{n}\right\}$ be a basis of $M_{\overline{1}}$, with multiplication defined by

$$
\begin{array}{r}
a . v_{i}=y_{i}=-v_{i} . a ; M . y_{i}=\{0\}, i=1, \ldots, n ; \\
v_{i} \cdot v_{j}=\delta_{i, j} a, i, j=1, \ldots, n ;
\end{array}
$$

and invariant scalar product given by

$$
\begin{array}{r}
B(a, a)=1 ; B\left(M_{1}, a\right)=\{0\}, \\
B\left(y_{i}, y_{j}\right)=0=B\left(v_{i}, v_{j}\right) ; B\left(y_{i}, v_{j}\right)=\delta_{i, j}=-B\left(v_{j}, y_{i}\right), i, j=1, \ldots, n ;
\end{array}
$$

Consider $\left\{m_{1}, m_{2}, \ldots m_{n}\right\} \subseteq K \backslash\{0\}$ and the operator $D \in O p_{a}(M)_{1}$ defined by $D(a)=\sum_{i=1}^{n} m_{i} y_{i} ; D\left(v_{i}\right)=m_{i} a$ and $D\left(y_{i}\right)=0$. Then the generalized double extension of $(M, B)$ by the one dimensional Malcev superalagbera $K e_{1}$ (by means of $D$ and a) is a quadratic Malcev superalgebra with reductive even part and the action of the even part on the odd part is not completely reducible.

In fact we have $\mathfrak{k}=\mathbb{K} e \oplus M \oplus \mathbb{K} e^{*}$ equipped with the even skew-symmetric bilinear map on $\mathfrak{k}$ defined by

$$
\begin{array}{r}
e e=a ; v_{i} v_{j}=\delta_{i, j} a ; e v_{i}=m_{i} a ; a e=\sum_{i=1}^{n} m_{i} y_{i}-e^{*}, \\
a v_{i}=y_{i}-m_{i} e^{*}, e^{* \mathfrak{k}}=y_{i} \mathfrak{k}=\{0\},
\end{array}
$$

Suppose now that $M_{1}$ is a completely reducible $M_{0}$-module. Then there is an $M_{0}$-submodule $X$ such that $M_{1}=X \oplus Y$ where $Y=<y, \ldots, y_{n}, e^{*}>$. But $M_{0} X \subseteq$ $M_{0} M_{1} \subseteq Y$ and $M_{0} X \subseteq X \cap Y=\{0\}$. As $M_{0} Y=\{0\}$ then $M_{0} M_{1}=\{0\}$ which is a contradiction.

Now we shall show the converse of the Theorem 2.3

Theorem 2.5. Let $\left(M=M_{\overline{0}} \oplus M_{\overline{1}}, B\right)$ be a B-irreducible quadratic Malcev superalgebra such that $\operatorname{dim} M>1$. If $\mathfrak{z}(M) \cap M_{\overline{1}} \neq\{0\}$ then $(M, B)$ is a generalized double extension of a quadratic Malcev superalgebra $(N, \widetilde{B})$ such that $\operatorname{dim} N=\operatorname{dim} M-2$ by the one-dimensional Malcev superalgebra with even part zero.

We will prove the result by showing the following sequence of claims as it is done in case of the quadratic Lie superalgebras [2, and in case of the odd-quadratic Lie superalgebras [3. First, we will determine the quadratic Malcev superalgebra $(N, \widetilde{B})$; then we will show that the quadratic Malcev superalgebra $(M, B)$ is the generalized double extension of $(N, \widetilde{B})$ by the one-dimensional Malcev superalgebra $(\mathbb{K} e)_{\overline{1}}$.

Proof. Let us assume that $(M, B)$ is a $B$-irreducible quadratic Malcev superalgebra such that $\operatorname{dim} M>1$ and $\mathfrak{z}(M) \cap M_{\overline{1}} \neq\{0\}$. We set $e^{*}$ a non-zero element of $\mathfrak{z}(M) \cap M_{\overline{1}}$ and denote $I=\mathbb{K} e^{*}$. As $B$ is even we have $M_{\overline{0}} \subseteq J$, where $J$ is the 
orthogonal of $I$ with respect to $B$. Since $B$ is non-degenerate and even then there exists $e \in M_{\overline{1}}$ such that $B\left(e^{*}, e\right) \neq 0$. We may assume that $B\left(e^{*}, e\right)=1$. As $e \notin J$ and $\operatorname{dim} J=\operatorname{dim} M-1$ we infer that $M=J \oplus \mathbb{K} e$. Consider the two-dimensional vector subspace $A=\mathbb{K} e^{*} \oplus \mathbb{K} e$ of $M$. Since $\left.B\right|_{A \times A}$ is non-degenerate we have $M=A \oplus N$, where $N$ is the orthogonal of $A$ with respect to $B$. It comes that $\widetilde{B}=\left.B\right|_{N \times N}$ is non-degenerate. As $B$ is even we have $\mathbb{K} e^{*} \subseteq J$, and so $\mathbb{K} e^{*} \oplus N \subseteq J$. From $\operatorname{dim}\left(\mathbb{K} e^{*} \oplus N\right)=\operatorname{dim} M-1=\operatorname{dim} J$ it comes that $J=\mathbb{K} e^{*} \oplus N$. So $N$ is a graded vector subspace of $M$ contained in the graded ideal $J=N \oplus \mathbb{K} e^{*}$ of $M$. Then we have

$$
X Y=\alpha(X, Y)+\varphi(X, Y) e^{*}, \quad \forall X, Y \in N,
$$

where $\alpha(X, Y) \in N$ and $\varphi(X, Y) \in \mathbb{K}$. Further,

$$
e X=D(X)+\psi(X) e^{*}, \quad \forall X \in N,
$$

where $D(X) \in N$ and $\psi(X) \in \mathbb{K}$.

Claim 1. Then $N$ endowed with the multiplication $\alpha$ is a Malcev superalgebra and $\widetilde{B}$ is an invariant scalar product on $N$.

Proof of Claim 1: From graded skew-symmetry on $M$ we get that

$$
\begin{array}{lll}
\alpha(X, Y)=-(-1)^{x y} \alpha(Y, X), & & \forall X \in N_{x}, Y \in N_{y}, \\
\varphi(X, Y)=-(-1)^{x y} \varphi(Y, X), & \forall_{X \in N_{x}, Y \in N_{y}} .
\end{array}
$$

Moreover, observing that for all $X \in N_{x}, Y \in N_{y}, Z \in N_{z}, T \in N_{t}$

$$
\begin{aligned}
& (X Z)(Y T)=\alpha(\alpha(X, Z), \alpha(Y, T))+\varphi(\alpha(X, z), \alpha(Y, T)) e^{*}, \\
& ((X Y) Z) T=\alpha(\alpha(\alpha(X, Y) Z) T)+\varphi(\alpha(\alpha(X, Y), Z), T) e^{*},
\end{aligned}
$$

by the second property of definition of Malcev superalgebras, it comes that for all $X \in N_{x}, Y \in N_{y}, Z \in N_{z}, T \in N_{t}$

$$
\begin{aligned}
(-1)^{y z} \alpha(\alpha(X, Z), \alpha(Y, T))= & \alpha(\alpha(\alpha(X, Y), Z), T) \\
& +(-1)^{x(y+z+t)} \alpha(\alpha(\alpha(Y, Z), T), X) \\
& +(-1)^{(x+y)(z+t)} \alpha(\alpha(\alpha(Z, T), X), Y) \\
& +(-1)^{t(x+y+z)} \alpha(\alpha(\alpha(T, X), Y), Z),
\end{aligned}
$$

and

$$
\begin{aligned}
(-1)^{y z} \varphi(\alpha(X, Z), \alpha(Y, T))= & \varphi(\alpha(\alpha(X, Y), Z), T) \\
& +(-1)^{x(y+z+t)} \varphi(\alpha(\alpha(Y, Z), T), X) \\
& +(-1)^{(x+y)(z+t)} \varphi(\alpha(\alpha(Z, T), X), Y) \\
& +(-1)^{t(x+y+z)} \varphi(\alpha(\alpha(T, X), Y), Z) .
\end{aligned}
$$

By (2.5) and (2.6) we infer that $N$ endowed with the multiplication $\alpha$ is a Malcev superalgebra and we denote $\alpha=(,)_{N}$. It is immediate that $\widetilde{B}$ is an invariant scalar product on $N$ completing the proof of Claim 1.

Since $e \in M_{\overline{1}}$ then $[e, e]$ is not necessarily zero, and we can write

$$
[e, e]=A_{0},
$$

where $A_{0} \in N_{\overline{0}}$. 
Claim 2. Then $D$ is an odd skew-supersymmetric Malcev operator of $(N, \widetilde{B})$ such that

$$
\begin{aligned}
& D^{2}\left(A_{0}\right)=\frac{1}{2}\left(A_{0} A_{0}\right)_{N} \\
& D\left(A_{0} X\right)=A_{0} D(X)-D\left(A_{0}\right) X, \\
& \left(A_{0} X\right) Y=D(D(X) Y)+D^{2}(X Y)+(-1)^{x} D(X) D(Y)+(-1)^{x y} D^{2}(Y) X, \\
& A_{0}(X Y)=D(D(X) Y)+D^{2}(X) Y-(-1)^{x y}\left\{D^{2}(Y) X+D(D(Y) X)\right\},
\end{aligned}
$$

for every $X \in M_{x}, Y \in M_{y}$. Moreover, $(M, B)$ is the generalized double extension of $(N, \widetilde{B})$ by the one-dimensional Malcev superalgebra $(\mathbb{K} e)_{\overline{1}}$ (by means of $D$ and $A_{0}$ ).

Proof of Claim 2: We start by proving that $D$ is an odd skew-supersymmetric Malcev operator of $(N, \widetilde{B})$. It is clear that $D$ is a homogeneous map of degree $\overline{1}$. The second property of definition of Malcev superalgebras for all $X \in N_{x}, Y \in N_{y}, Z \in N_{z}$

$$
\begin{aligned}
(-1)^{y z}(X Z)(Y e)= & ((X Y) Z) e+(-1)^{x(y+z+1)}((Y Z) e) X \\
& +(-1)^{(x+y)(z+1)}((Z e) X) Y+(-1)^{x+y+z}((e X) Y) Z,
\end{aligned}
$$

implies that $D$ is a Malcev operator of $N$. On the other hand, Using the invariance of $B, B([e, X], Y)=-(-1)^{x} B(X,[e, Y])$, for every $X \in N_{x}, Y \in N$, we obtain

$$
\widetilde{B}(D(X), Y)=-(-1)^{x} \widetilde{B}(X, D(Y)), \quad \forall X \in N_{x}, Y \in N,
$$

which means that $D \in\left(O p_{a}(N, \widetilde{B})\right)_{\overline{1}}$. Due to $-(e e)(e e)=-2((e e) e) e$ we obtain $D^{2}\left(A_{0}\right)=\frac{1}{2}\left(A_{0} A_{0}\right)_{N}$. From $B([e, X], Y)=B(e,[X, Y])$, for all $X, Y \in N$, we get that

$$
\varphi(X, Y)=-B(D(X), Y), \quad \forall X, Y \in N .
$$

Due to $B([e, X], e)=B(e,[X, e])$, for all $X \in N_{x}$, we conclude that

$$
\psi(X)=-(-1)^{x} B\left(X, A_{0}\right), \quad \forall X \in N_{x} .
$$

Using sucessively the second property of definition of Malcev superalgebras

$$
\begin{aligned}
-(e e)(e X)= & ((e e) e) X+(-1)^{x}((e e) X) e+((e X) e) e \\
& +(-1)^{x}((X e) e) e \\
(-1)^{x}(e X)(e Y)= & ((e e) X) Y-(-1)^{x+y}((e X) Y) e+((X Y) e) e \\
& +(-1)^{y x}((Y e) e) X \\
(-1)^{x}(e e)(X Y)= & ((e X) e) Y-(-1)^{x+y}((X e) Y) e \\
+ & +(-1)^{(1+x)(1+y)}((e Y) e) X+(-1)^{y x}((Y e) X) e
\end{aligned}
$$

for $X \in M_{x}, Y \in M_{y}$, we infer respectively assertions (2.7), (2.8), and (2.9), which states Claim 2.

Using the results of Claims 1 and 2 we conclude Theorem 2.5.

\section{Structure and inductive Classification of QuAdratic Malcev SUPERALGEBRAS WITH REDUCTIVE EVEN PART}

Theorem 3.1. (proved in [4]) Let $\left(M=M_{\overline{0}} \oplus M_{\overline{1}}, B\right)$ be a B-irreducible quadratic Malcev superalgebra such that $\operatorname{dim} M>1$. If $\mathfrak{z}(M) \cap M_{\overline{0}} \neq\{0\}$ then $(M, B)$ is a double extension of a quadratic Malcev superalgebra $(N, \widetilde{B})$ such that $\operatorname{dim} N=$ $\operatorname{dim} M-2$ by the one-dimensional Lie algebra. 
So to conclude our study we must analyse the case where $M$ is a $B$-irreducible quadratic Malcev superalgebra with reductive even part such that $\mathfrak{z}(M)=\{0\}$. Analogously to the Lie case (cf. [2]), the socle of $M$ has a remarkable importance in this study and we can prove with the same reasoning that if $M$ is a $B$-irreducible quadratic Malcev superalgebra with reductive even part that is neither the one dimensional Lie algebra nor a simple Malcev superalgebra the socle coincides with the center. As a consequence of this result, with a proof analogously to the one in Lie case [2], we characterize quadratic Malcev superalgebras with reductive even part that have null center as semisimple Malcev superalgebras.

Finally we present an inductive description of quadratic Malcev superalgebras such that the even part is a reductive Malcev algebra. Clearly we improve the results proved in 4 since we work with a larger class of superalgebras.

Let $\mathfrak{U}$ be the set formed by $\{0\}$, basic classical Lie superalgebras, simple (non Lie) Malcev algebra and one-dimensional Lie algebra.

Theorem 3.2. Let $\left(M=M_{\overline{0}} \oplus M_{\overline{1}}, B\right)$ be a quadratic Malcev superalgebra such that $M_{\overline{0}}$ is a reductive Malcev algebra. Then either $M$ is an element of $\mathfrak{U}$, or $M$ is obtained by a sequence of double extensions by the one-dimensional Lie algebra, or generalized double extensions by the one-dimensional Malcev superalgebra with even part zero, and/or by orthogonal direct sums of quadratic Malcev superalgebras from a finite number of elements of $\mathfrak{U}$.

Proof. The proof is by induction on the dimension of $M$ and is analogous to the corresponding theorem in Lie case (cf. 2]), using the fact that a simple quadratic Malcev superalgebra is either a basic classical Lie superalgebra or is the simple (non Lie) Malcev algebra.

\section{REFERENCES}

11] H. Albuquerque, Contribuições para a teoria das superálgebras de Malcev, Doctoral thesis, University of Coimbra, 1993.

[2] H. Albuquerque, E. Barreiro, and S. Benayadi, Quadratic Lie superalgebras with reductive even part, Pré-Publicações do Departamento de Matemática-Universidade de Coimbra, preprint number 07-08.

[3] H. Albuquerque, E. Barreiro, and S. Benayadi, Odd-quadratic Lie superalgebras, PréPublicações do Departamento de Matemática-Universidade de Coimbra, preprint number 07-09.

[4] H. Albuquerque and S. Benayadi, Quadratic Malcev superalgebras, J. Pure Appl. Algebra 187 (2004), 19-45.

[5] I. Bajo, S. Benayadi, and M. Bordemann, Generalized double extension and description of quadratic Lie superalgebras, in preparation.

[6] H. Benamor and S. Benayadi, Double extension of quadratic Lie superalgebras, Comm. Algebra 27 (1999), 67-88.

[7] S. Benayadi, Quadratic Lie superalgebras with the completely reducible action of the even part on the odd part, J. Algebra 223 (2000), 344-366.

[8] _ Inductive classification of quadratic Lie superalgebras, Res. Exp. Math. 25 (2002), $135-148$.

[9] Socle and some invariants of quadratic Lie superalgebras, J. Algebra 261 (2003), 245-291.

[10] M. Bordemann, Nondegenerate invariant bilinear forms on nonassociative algebras, Acta Math. Univ. Comenian. LXVI (1997), 151-201.

[11] A. Elduque, Lie superalgebras with semisimple even part, J. Algebra 138 (1996), 649-663.

[12] A. Medina and P. Revoy, Algèbres de Lie et produit scalaire invariant, Ann. Sci. École. Norm. Sup. (4) 18 (1985), 553-561.

[13] M. Scheunert, The Theory of Lie Superalgebras, Lectures Notes in Mathematics, vol. 716, Springer-Verlag Berlin Heidelberg, 1979. 
Helena Albuquerque, Departamento de Matemática, Universidade de Coimbra, Apartado 3008, 3001-454 COIMBRA, PORTUGAL E-mail address: LENA@MAT.uc.PT

Elisabete Barreiro, Departamento de Matemática, Universidade de Coimbra, Apartado 3008, 3001-454 CoImbra, PORTUGAL E-mail address: мEFB@mat.uc. PT

Saïd Benayadi, Laboratoire de Mathmatiques et Applications de Metz, CNRS-UMR 7122, Universit Paul Verlaine, Ile du Saulcy, 57045 Metz Cedex 1, FRANCE E-mail address: BENAYADI@PONCELET. UNIV-METZ.FR 